\title{
Proof-of-Concept Energy-Efficient and Real-Time Hemodynamic Feature Extraction from Bioimpedance Signals using a Mixed- Signal Field Programmable Analog Array
}

\author{
Hakan Töreyin, Member, IEEE, Sahil Shah, Student Member, IEEE, Sinan Hersek, Omer T. Inan, \\ Senior Member, IEEE, and Jennifer Hasler, Senior Member, IEEE
}

\begin{abstract}
We present a mixed-signal system for extracting hemodynamic parameters in real-time from noisy electrical bioimpedance (EBI) measurements in an energy-efficient manner. The proof-of-concept system consists of floating-gatebased analog signal processing (ASP) electronics implemented on a field programmable analog array (FPAA) chip interfaced with an on-chip low-power microcontroller. Physiological features important for calculating hemodynamic parameters (e.g., heart rate, blood volume, and blood flow) are extracted using the custom signal processing circuitry, which consumes a total power of $209 \mathrm{nW}$. Testing of the signal processing circuitry has been performed using $\sim \mathbf{5 8 0}$ sec of an impedance plethysmography dataset collected from the knee of a subject using a custom analog EBI front-end. Results show the similarities of variations in heart rate, blood volume, and blood flow calculated using features extracted by the ASP circuitry implemented on an FPAA and a MATLAB digital signal processing algorithm.
\end{abstract}

\section{INTRODUCTION}

Electrical properties (i.e., conductivity and permittivity) of biological tissues are unique to the type of tissue (e.g., blood, fat, bone) [1]. Therefore, based on tissue electrical properties, the composition of a tissue (e.g., fat, bone, muscle) and changes in the tissue content (e.g., associated with edema, fluid accumulation) can be monitored. EBI measurement is a technique used to obtain these properties of biological tissues by injecting a small-magnitude of ac current to a tissue under investigation and measuring the resulting potential difference across the tissue. It is widely used in research for many applications ranging from extracting tissue composition of a body part (e.g., fat composition [2]) to hemodynamic monitoring (e.g., cardiac output [3]), since EBI is non-invasive in nature and has very high temporal-resolution. Additionally, EBI can be measured using low-cost electronics, thereby making EBI a potentially feasible biosignal modality for health assessment applications outside the clinic [4].

Conventionally, EBI systems consist of a front-end block that interfaces with the tissue via electrodes [5] and a digital block for streaming and logging the bioimpedance data to

This material is based upon work supported in part by the Defense Advanced Research Projects Agency (DARPA), Arlington, VA under Contract No. W911NF-14-C-0058.

H. Töreyin was with Georgia Institute of Technology, Atlanta, Georgia 30332. He is now with the Department of Electrical and Electronics Engineering, Bilkent University, Turkey.

S. Shah, S. Hersek, O. T. Inan, and J. Hasler are with the School of Electrical and Computer Engineering, Georgia Institute of Technology, Atlanta, Georgia 30332 \{oeinan@gmail.com,jenniolson@gmail.com\}.

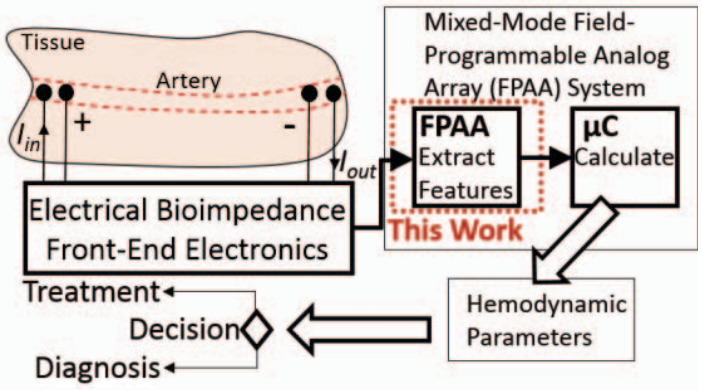

Fig. 1. An energy-efficient signal processing circuitry implemented on a field-programmable analog array performs real-time extraction of heart rate (HR) as well as blood volume (BV) and blood flow (BF) variables; from EBI measurements from a knee.

later extract physiologically relevant information (e.g., heart rate, blood flow). While a system approach as such is convenient for systems designed for use in clinical settings, for closed-loop wearable medical applications combining health monitoring and treatment or systems designed for use in resource-limited settings (e.g., during outdoor activities), an EBI system needs to extract physiological information in real-time while not compromising accuracy, energyefficiency, or portability.

Accordingly, we present custom-designed and low-power signal processing circuitry extracting hemodynamics parameters from impedance plethysmography (IPG) signals, which reflect changes in blood volume and are obtained from EBI measurements. To benefit from the power and area efficiency of analog computations while making use of the accuracy of digital, our approach performs computations in the mixed-signal domain, where a floating-gate CMOS based custom analog-signal-processing circuitry and a digital processor (i.e., processor of a laptop computer) perform the feature extraction and calculations, respectively. For the ASP circuitry, an FPAA implementation is preferred over a custom analog design, as the FPAA serves as a flexible silicon fabric where other circuitry corresponding to the remaining components of the ultimate system presented in Fig. 1 would also be implemented. In the ultimate system, the FPAA would also enable tuning the circuit through floatinggate programming to implement subject-dependency and adaptive decision-making.

The organization of this paper is as follows. In Section II, the EBI front-end and the FPAA architectures are summarized. Important features of IPG signals in regards of hemodynamic parameters and the analog circuitry are detailed in Section III. Measurements and results from the 


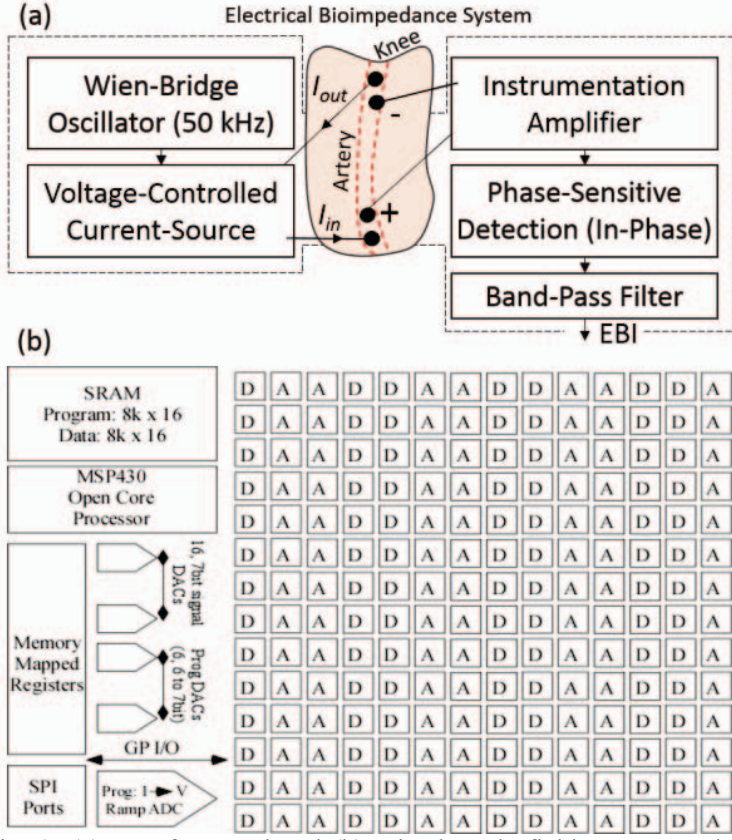

Fig. 2. (a) EBI front-end and (b) mixed-mode field-programmable analog array (FPAA) systems.

custom signal processing circuitry as well as comparison with off-line processing results are presented in Section IV. The paper is concluded in Section V.

\section{EBI FRONT-END AND FPAA}

\section{A. EBI Front-End}

Bioimpedance measurements are achieved by injecting a small-magnitude ac current to a tissue and measuring the resulting potential difference. The current is generated via a Wien-Bridge oscillator that generates a $50 \mathrm{kHz}$ sinusoidal signal and feeds into a high output-impedance voltagecontrolled current-source delivering the current to the tissue through two electrodes (current electrodes). The frequency of $50 \mathrm{kHz}$ ensures that current could propagate through both intra- and extra-cellular fluid of the tissue. The potential across the tissue is measured via two other electrodes (voltage electrodes) to minimize the effect of skin-electrode impedance and amplified by an instrumentation amplifier. To obtain the resistance component of the impedance, a phasesensitive detection circuitry is designed using an analog switch controlled by a clock that is in-phase with the injected current. The ac component of the resistance, namely the impedance plethysmography (IPG) signal that reflects the changes in blood volume, is then obtained using a band-pass filter (BW: $0.1 \mathrm{~Hz}-20 \mathrm{~Hz}$ ). For more details on the IPG circuitry, the reader is referred to [5].

\section{B. Mixed-Mode Field-Programmable Analog Array}

The FPAA is a mixed signal large scale SoC described in detail here [6]. Floating gate based FPAA allows for programmability and re-configurability, usually only offered by digital FPGA. Due to these features they have an ability to operate beyond the energy efficiency wall, seen in a general digital signal processor.

The block diagram of the FPAA used in this work is presented in Fig. 2(b). In the figure, A stands for

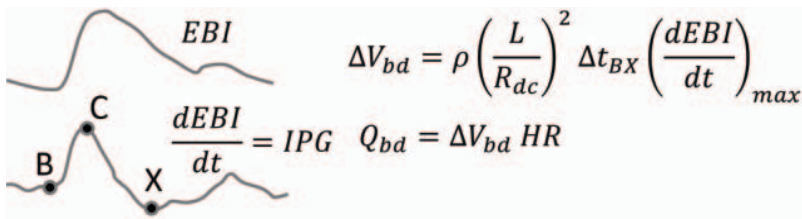

Fig. 3. Example EBI and $d E B I / d t$, namely IPG, signals (redrawn from [5]) with features important for extracting hemodynamic parameters highlighted. Pulsatile blood volume, $\Delta V_{b d}$, is proportional to the timevarying parameters of (i) the time difference between the points $B$ and $X$, namely $\Delta t_{B X}$ and (ii) the peak-to-peak amplitude (i.e., magnitude difference of points $C$ and $B$ ) of the rising portion of the IPG signal, namely $(d E B I / d t)_{\max }$, and the constants of (iii) resistivity of blood, namely $\rho$, the distance between the voltage electrodes, and the dc resistance of the tissue, namely $R_{d c}$. The blood flow rate, namely $Q_{b d}$ is the product of $\Delta V_{b d}$ and the heart rate (HR).

Computational Analog Blocks (CAB) whereas D stands for Computational Logic Blocks (CLB). The CABs and CLBs are interconnected in Manhattan style routing using $\mathrm{C}$ (Connection) and Switch (S) blocks. Interconnects are composed of array of floating gate transistors which are programmed using hot-electron injection and globally erased using Fowler-Nordheim tunneling. Target and Switch programming are discussed in detail somewhere else [7]. A $\mathrm{CAB}$ consists of analog elements such as an Operational Transconductance Amplifier (OTA), Transmission Gates (Tgate), capacitors and current mirrors. As a part of the infrastructure the FPAA consists of 16 7-bit DACs which are used for generating signals. It also consists of two 7-bit ADCs and a 14-bit ramp ADC for data acquisition. They are controlled using an open source MSP430 which writes and reads data from $8 \mathrm{k}$ x 16 data memory.

\section{ASP CIRCUIT FOR FEATURE EXTRACTION}

\section{A. Important Features of IPG for Hemodynamic Monitoring}

The IPG signal is widely used for hemodynamic monitoring, and can be used to track variations in blood volume as a function of time. Synchronous with the heartbeat, the IPG signal is also used to calculate heart rate. Lastly, from the product of blood volume and heart rate, blood flow is calculated [8-9].

An example EBI waveform, its first time-derivative (i.e., IPG) and the critical features of IPG described in [10] are presented in Fig. 3. In [9], IPG features are related to pulsatile blood volume (i.e., $\Delta V_{b d}$ ) and blood flow (i.e., $Q_{b d}$ ) using equations presented in Fig. 3. The variables of the equations are described in the Fig. 3 caption.

\section{B. ASP Circuitry for Feature Extraction}

The signal processing circuitry is designed to first identify the critical features, points $B, C$, and $X$ in IPG, in an energyefficient manner and then deliver the features to a processor to accurately calculate heart rate, blood volume, and blood flow.

First, the signal-processing circuitry (Fig. 4) smooths the noisy EBI signal using a low-pass filter with a cutoff frequency of $f_{-3 d B}=3 \mathrm{~Hz}$, which is implemented as a firstorder $\mathrm{Gm}-\mathrm{C}$ stage using a 9-transistor OTA. The first timederivative of the smoothed-EBI signal is obtained using a $\mathrm{C}^{4}$ band-pass filter with a center frequency $\left(f_{-3 d B, \text { Center }}=3.5 \mathrm{~Hz}\right)$ set to a value greater than the 


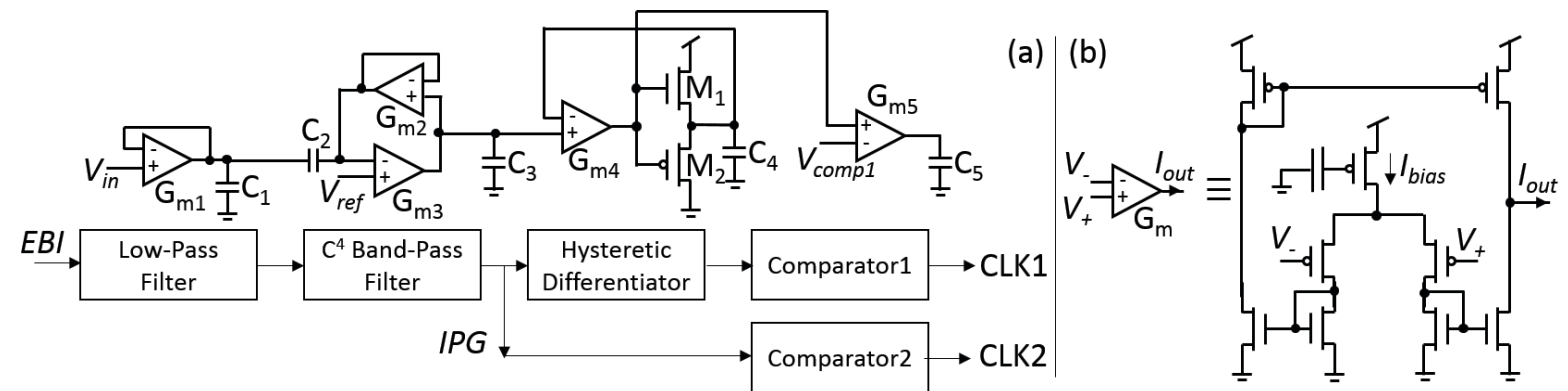

Fig. 4. IPG signal processing circuit. (a) The low-pass filter is used to generate a smooth version of the IPG signal, from which the $1^{\text {st }}$ derivative is obtained using a $\mathrm{C}^{4}$ band-pass filter. A hysteretic differentiator is implemented to identify the two minimum values from the $1^{\text {st }}$ derivative of the IPG signal. The comparator-1 at the output of the hysteretic differentiator generates the CLK1, which serves as the enable signal for running the ADC controlled by the microcontroller. (b) $\mathrm{G}_{\mathrm{m}}$ stages are implemented as 9-transistor OTAs, where the bias current is set by floating-gate pMOS devices.

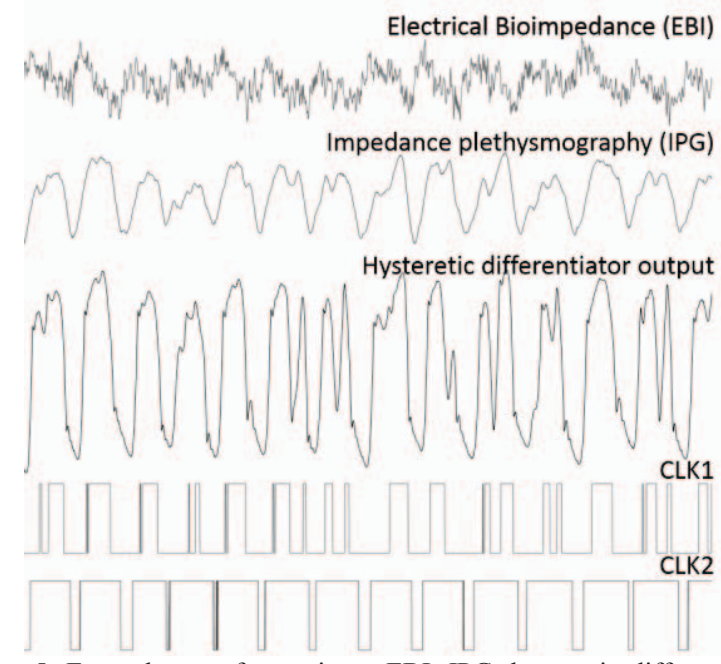

Fig. 5. Example waveforms; input EBI, IPG, hysteretic differentiator output, and CLK1 and CLK2. Data duration: $10 \mathrm{sec}$.

fundamental frequency of the EBI signal ( $\mathrm{f} \sim 1 \mathrm{~Hz}$ ), thereby differentiating the EBI signal. To compensate for the reduction in gain, the gain is set to $\sim 11 \mathrm{~dB}$ by increasing the load capacitance, $\mathrm{C}_{3}$. To identify the points $B$ and $X$, the timederivative signal, namely IPG, is fed to a hysteretic differentiator, output of which changes abruptly when there is a significant change in the IPG [11]. For small changes in the IPG however, the output almost does not change, thereby inherently suppressing the noisy behavior of the IPG. The hysteretic differentiator output is then converted into a clock, namely CLK1, which is generated to determine one of the time instances (point $B$ ) when the microprocessor digitizes the IPG and to calculate the time difference between the two time instances (points $B$ and $X$ ). The signal processing circuitry generates another clock, CLK2, directly from the output of $\mathrm{C}^{4} \mathrm{BPF}$, to determine the second time instance (point $C$ ) when the microprocessor digitizes the IPG. Both clocks are generated using comparators implemented as open-loop OTA amplifiers with reference voltages, $V_{\text {compl }}$ and $V_{\text {comp 2. }}$ In Fig. 4, only the comparator schematic corresponding to CLK1 is shown. Example waveforms from the circuit and the resultant clocks are presented in Fig. 5.

\section{RESUlts, Discussion, AND FUtURE WORK}

The ASP circuitry implemented on the FPAA has been fed with $\sim 580 \mathrm{sec}$. of EBI data, which have been collected from the knee of a healthy subject. The data was collected using the analog front-end described in Section II and a
BioNomadix (Biopac Systems Inc., Goleta, CA). The study was approved by the GT Institutional Review Board (IRB) and the Army Human Research Protection Office (AHRPO). Wet-gel electrodes have been used to improve the skinelectrode impedance and data was collected when the subject was seated and legs extended.

For the bias values used, power consumptions are calculated as $0.12 \mathrm{nW}, 7.6 \mathrm{nW}, 1.25 \mathrm{nW}$, and $200 \mathrm{nW}$ for the LPF, $\mathrm{C}^{4} \mathrm{BPF}$, hysteretic differentiator, and the comparators, respectively, making a total power consumption of $\sim 209 \mathrm{nW}$. In its current form, the FPAA board is powered from the USB port. In an ultimate wearable design where the FPAA is powered by a lightweight $3.7 \mathrm{~V} 1000 \mathrm{mAh}$ battery, the battery could power the feature-extraction circuitry and the on-board processor (estimated power consumption when the processor is never put into sleep mode: $\sim 12 \mathrm{~mW}$ ) for 8 days.

For validation of the ASP circuitry, MATLAB is used to calculate the heart rate (HR), blood volume variable (BVV), which is defined as the product of $\Delta t_{B X}$ and $(d E B I / d t)_{\max }$ in Fig. 3, and the blood flow variable (BFV), which is defined as the product of blood volume variable and heart rate; as well as visualize the results. The high-to-low and low-to-high transitions of the CLK1 correspond to the points $B$ and $X$, respectively. The point $C$ is where IPG is at its maximum when the CLK2 is low. For comparison with digital signal processing, a MATLAB code performing; (i) time-derivation of the raw IPG signal smoothed using a Savitzky-Golay Filter of $2^{\text {nd }}$ order and 201 taps, (ii) identification of the local maxima (points $C$ ) and minima ( $B$ and $X$ ), and (iii) calculation of the HR, BVV, and BFV; is generated. In Fig. 6(a), a portion of the IPG signal obtained from the FPAA and MATLAB as well as a portion of the FPAA IPG data with points $B, C$, and $X$ labeled are presented. HR, BVV, and BFV calculated using $B-C-X$ features obtained from the FPAA and the MATLAB code are presented in Fig. 6(b). The smoothed versions of the datasets using a Savitzky-Golay filter of $2^{\text {nd }}$ order and 51 taps show that datasets from the ASP circuitry and MATLAB display similar trends over the course of the measurement.

The functions implemented on MATLAB for proof-ofconcept purposes can also be implemented on the MSP430 microcontroller. In an exemplary algorithm for such implementation in Fig. 7, the clocks CLK1 and CLK2 are fed to the MSP430 using general purpose I/Os. A counter is used to measure the time between two peaks and the absolute value of the signal is measured using a 14-bit ramp ADC 

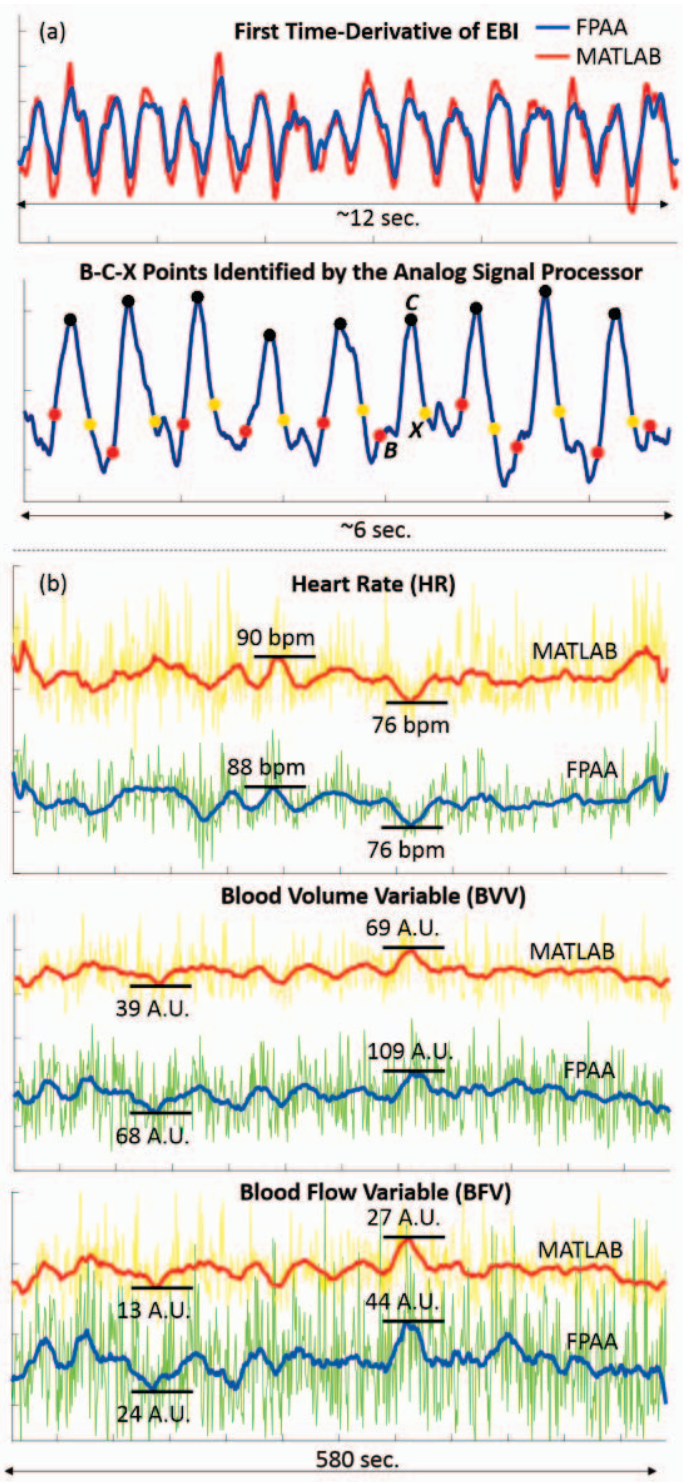

Fig. 6. (a) Comparison of the time-derivative IPG signals obtained from the ASP implemented on the FPAA and digital implementation on MATLAB. The points $B-C-X$ on the IPG signal of the FPAA are shown. (b) Variation of heart rate, and blood volume and blood flow variables obtained using the ASP presented and the MATLAB implementation display similar trends over the course of a $580 \mathrm{sec}$. IPG measurement.

having a sampling rate of 200 Sps. The MSP430 could continuously monitor the general purpose $\mathrm{I} / \mathrm{O}$ as in the case of Fig. 7, and alternatively this could be interrupt driven. The counter and the ADC values are stored on to the SRAM. For variation trends, the values could be averaged over several periods of IPG. Secondly, in current work the IPG signal is streamed to the FPAA using a data acquisition device because of the constraints of on-chip memory. Ultimately, the voltage electrodes could be fed directly to the FPAA and filtered on-chip using an LNA and a band pass filter. Thirdly, validation of the method with the study of people with diagnosed hemodynamic pathologies is left as future work.

\section{CONCLUSION}

In this study, a custom ASP circuitry extracting features of an IPG signal to calculate hemodynamic parameters, is

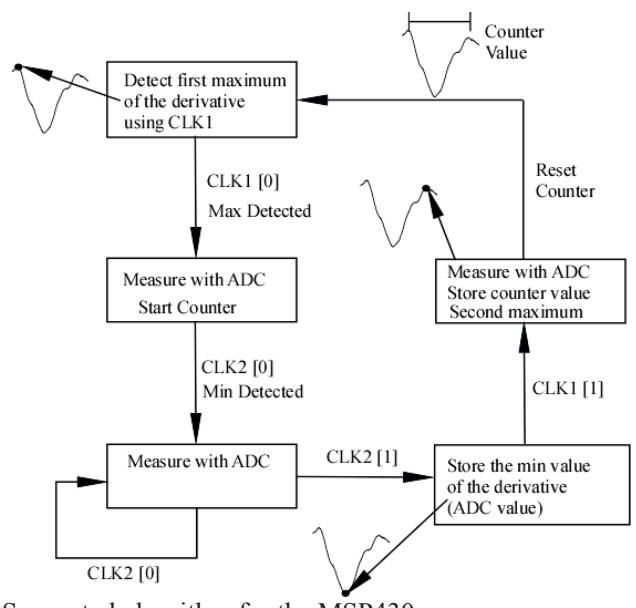

Fig. 7. Suggested algorithm for the MSP430.

presented. The circuitry generates and delivers a processor the IPG data and digital clocks providing the timing information of when to digitize the IPG, and start/stop a counter for calculating time difference between features within a heartbeat for pulsatile blood volume and flow calculations. The clocks are also used to calculate the time difference from beat to beat for heart rate calculation. Based on the promising initial measurement results, we envision the circuitry being an energy-efficient solution for processing IPG signals in wearable health-monitoring applications.

\section{REFERENCES}

[1] S. Gabriel, R. W. Lau, and C. Gabriel. "The dielectric properties of biological tissues: II. Measurements in the frequency range $10 \mathrm{~Hz}$ to 20 GHz." Physics in medicine and biology, vol. 41, no. 11, pp. 22512269, 1996.

[2] Nyboer, J. Electrical Impedance Plethysmograph, 2nd ed.; Thomas, C., Ed.; Thomas publishers: Springfield, IL, USA, 1970.

[3] Cotter G, et al. "Accurate, Noninvasive Continuous Monitoring Of Cardiac Output By Whole-Body Electrical Bioimpedance" Chest 2004;125(4):1431-1440.

[4] Y. L. Zheng, et al. "Unobtrusive sensing and wearable devices for health informatics." IEEE Transactions on Biomedical Engineering, vol. 61, no. 5, pp. 1538-1554, 2014.

[5] S. Hersek, H. Töreyin, and O. T. Inan. "A Robust System for Longitudinal Knee Joint Edema and Blood Flow Assessment Based on Vector Bioimpedance Measurements." IEEE Transactions on biomedical circuits and systems, vol. 10, no. 3 (2016): 545-555.

[6] S. George, et al., "A programmable and configurable mixed-mode FPAA SoC," IEEE Transactions on Very Large Scale Integration (VLSI) Systems, vol. 24, no. 6, pp. 2253-2261, June 2016.

[7] S. Kim, J. Hasler, and S. George, "Integrated floating-gate programming environment for system-level ICs," IEEE Transactions on Very Large Scale Integration (VLSI) Systems, vol. PP, no. 99, pp. $1-9,2016$.

[8] J. Nyboer, M. M. Kreider, and L. Hannapel, "Electrical impedance plethysmography a physical and physiologic approach to peripheral vascular study," Circulation, vol. 2, pp. 811-821, 1950.

[9] W. G. Kubicek, R. P. Patterson, and D. A. Witsoe, "Impedance cardiography as a noninvasive method of monitoring cardiac function and other parameters of the cardiovascular system," Ann. New York Acad. Sci., vol. 170, pp. 724-732, 1970.

[10] A. Sherwood, M. T. Allen, J. Fahrenberg, R. M. Kelsey, W. R. Lovallo, and L. J. P. v. Doomen, "Methodological guidelines for impedance cardiography," Psychophysiol., vol. 27, pp. 1-23, 1990.

[11] C. Mead, Analog VLSI and Neural Systems, pp. 173-178, 1989, Addison-Wesley. 